This item was submitted to Loughborough's Research Repository by the author.

Items in Figshare are protected by copyright, with all rights reserved, unless otherwise indicated.

\title{
Lyapunov stability of charge transport in miniband semiconductor
} superlattices

\section{PLEASE CITE THE PUBLISHED VERSION}

http://dx.doi.org/10.1103/PhysRevB.88.165304

\section{PUBLISHER}

(C) American Physical Society

\section{VERSION}

VoR (Version of Record)

\section{PUBLISHER STATEMENT}

This work is made available according to the conditions of the Creative Commons Attribution-NonCommercialNoDerivatives 4.0 International (CC BY-NC-ND 4.0) licence. Full details of this licence are available at: https://creativecommons.org/licenses/by-nc-nd/4.0/

\section{LICENCE}

CC BY-NC-ND 4.0

\section{REPOSITORY RECORD}

Koronovskii, Alexey A., Alexander E. Hramov, V.A. Maximenko, Olga I. Moskalenko, Kirill N. Alekseev, M.T. Greenaway, T.M. Fromhold, and Alexander G. Balanov. 2019. "Lyapunov Stability of Charge Transport in Miniband Semiconductor Superlattices”. figshare. https://hdl.handle.net/2134/17296. 


\title{
Lyapunov stability of charge transport in miniband semiconductor superlattices
}

\author{
A. A. Koronovskii, ${ }^{1,2}$ A. E. Hramov, ${ }^{1,2}$ V. A. Maximenko, ${ }^{1,2}$ O. I. Moskalenko,,${ }^{1,2}$ K. N. Alekseev, ${ }^{3}$ M. T. Greenaway, ${ }^{4}$ \\ T. M. Fromhold, ${ }^{4}$ and A. G. Balanov ${ }^{1,3}$ \\ ${ }^{1}$ Faculty of Nonlinear Processes, Saratov State University, Astrakhanskaya 83, Saratov, 410012, Russia \\ ${ }^{2}$ Saratov State Technical University, Politechnicheskaja 77, Saratov, 410054, Russia \\ ${ }^{3}$ Department of Physics, Loughborough University, Loughborough LE11 3TU, United Kingdom \\ ${ }^{4}$ School of Physics and Astronomy, University of Nottingham, Nottingham NG7 2RD, United Kingdom
}

(Received 18 December 2012; published 9 October 2013)

\begin{abstract}
We discuss a numerical method for the calculation of the spectrum of Lyapunov exponents for spatially extended systems described by coupled Poisson and continuity equations. This approach was applied to the model of collective charge transport in semiconductor superlattices operating in the miniband transport regime. The method is in very good agreement with analytical results obtained for the steady state. As an illustrative example, we consider the collective electron dynamics in the superlattice subjected to an ac voltage and a tilted magnetic field, and conclusively show that, depending on the field parameters, the dynamics can exhibit periodic, quasiperiodic, or chaotic behavior.
\end{abstract}

DOI: 10.1103/PhysRevB.88.165304

PACS number(s): 73.21.-b, 05.45.-a

\section{INTRODUCTION}

Models based on coupled Poisson and continuity equations are often used to describe various phenomena in solid-state and plasma physics, where time evolution and spatial distribution of electric field is coupled self-consistently with the charge. ${ }^{1-7}$ Such systems can demonstrate a variety of dynamical behavior, including deterministic chaos. ${ }^{5,8-10}$ However, stability analysis (even numerical) of the systems away from the equilibrium still remains a nontrivial problem. The difficulty arises because the Poisson and continuity equations are both spatially extended, i.e., have infinitely dimensional phase space. In contrast, the majority of the existing techniques were developed for dynamical systems of finite dimension and concentrated parameters. Among the conventional tools of stability analysis, perhaps the most powerful and universal is calculation of the Lyapunov exponents. ${ }^{11}$ A positive value of the maximal Lyapunov exponent is a robust indicator of chaos, and the knowledge of other Lyapunov exponents provides more detailed information about the dynamics and the instabilities developed in the system. For example, quasiperiodic dynamics is characterized by two or more zero Lyapunov exponents, whereas hyperchaos features more than one positive Lyapunov exponent. Therefore, identification and stability analysis of such dynamical regimes require the study of the full spectrum of Lyapunov exponents.

Nowadays, the theory and techniques for the estimation of the Lyapunov exponents for the low-dimensional dynamical systems described by ordinary differential equations (ODEs) are quite well established. ${ }^{11-14}$ However, a straightforward application of these methods to the spatially extended systems is only possible for the systems which are naturally discrete in space, ${ }^{15-17}$ e.g., to the arrays of coupled oscillators or maps. Importantly, the direct application of numerical techniques for the calculation of Lyapunov exponents, developed for systems with finite dimension of the phase space, to continuous spatially extended systems is rather ambiguous ${ }^{17}$ and unreliable. ${ }^{15,18}$ The primary reason for this is that a perturbation of a solution of an extended system is determined not by a phase vector, but by a set of functions which depend on spatial coordinates, requiring a modification of the orthogonalization and normalization procedures involved in the Lyapunov exponents calculations. The above factors require a development of specific approaches to stability analysis of various spatially extended systems. ${ }^{16,19-22}$

In this paper, we propose an approach for the calculation of the spectrum of Lyapunov exponents for a system of coupled Poisson and continuity equations, and apply this method to a strongly coupled semiconductor superlattice (SL) operating in the miniband transport regime. ${ }^{23,24} \mathrm{We}$ should note that for weakly coupled SLs, in which the resonant tunneling transport mechanism dominates, the charge dynamics can be described by a spatially discrete version of the Poisson and continuity equations. ${ }^{25}$ In this case, the model is represented by a set of ODEs, whose number corresponds to the number of periods in the SL. Lyapunov stability analysis of such a spatially discrete model was discussed in Refs. 26 and 27. For a miniband SL described by partial differential equations (PDEs), application of the approaches developed for ODEs is neither straightforward nor apparent from the viewpoint of physics. ${ }^{28}$ Therefore, until now Lyapunov stability of the charge transport in minband SLs has been limited by the estimation of the largest Lyapunov exponent from time series. $^{28}$ With this, recent experimental works ${ }^{29-32}$ report on high-frequency mixing and highly nonlinear charge transport, which are apparently accompanied by complex spatially temporal dynamics of charge in miniband heavily doped SLs. Understanding of these dynamical regimes and instabilities associated with them in the appropriate models is important for further development and new design of the SL devices. However, their theoretical analysis is seriously limited by the shortage of reliable numerical tools allowing one to calculate the spectrum of Lyapunov exponents.

The technique we present here is a mathematically justified approach to the calculation of the spectrum of Lyapunov exponents in spatially extended Poisson and continuity equations. It has a clear physical interpretation and can be used for the identification and stability analysis of various types of dynamical regimes, including steady-state, periodic, quasiperiodic, and chaotic dynamics. 
We apply this method to generic exemplary models describing charge dynamics in both dc-biased SLs and SLs subjected to an ac voltage and a tilted magnetic field. Thus, the models considered in this paper take into account application of various fields used in a range of experimental ${ }^{29-33}$ and theoretical works. ${ }^{6,34,35}$ We analytically and numerically show that when the electron transport in the SL is static, the method demonstrates good agreement with the $n L$ criterion, conventionally used for the prediction of electric domain instability. ${ }^{36}$ We also show that the application of an ac voltage and a tilted magnetic field to the SL results in a variety of periodic, quasiperiodic, and even chaotic spatiotemporal charge dynamics.

The paper has the following structure. In Sec. II, we discuss the model describing the collective dynamics of charge in a SL. A general formalism related to a numerical estimation of the spectrum of Lyapunov exponents is given in Sec. III. In Sec. IV, we present the analytical results obtained in the steady state. In Sec. V, we apply the method to the characterization of different dynamical regimes in a SL with an applied ac voltage and tilted magnetic field. Finally, in Sec. VI, we summarize our results and outline further interesting applications of our method.

\section{MODEL OF A STRONGLY COUPLED SEMICONDUCTOR SUPERLATTICE}

Semiconductor superlattices (SLs), formed from nanolayers of different types of semiconductor materials, are of great interest for both fundamental and applied physics because of their unique quantum-mechanical properties and great potential for sub- $\mathrm{THz}$ and $\mathrm{THz}$ electronics. ${ }^{23-25,37}$ They are able to demonstrate negative differential conductance, ${ }^{23,37}$ which induces new regimes of collective charge transport accompanied by rich instability phenomena. $5,6,38-42$

The collective dynamics of miniband electrons ${ }^{6,25}$ can be described by the one-dimensional (1D) current continuity equation

$$
\frac{\partial n^{\prime}}{\partial t^{\prime}}=-\frac{1}{e} \frac{\partial J^{\prime}}{\partial x^{\prime}}
$$

and 1D Poisson equation

$$
\frac{\partial F^{\prime}}{\partial x^{\prime}}=\frac{e}{\varepsilon_{0} \varepsilon_{r}}\left(n^{\prime}-n_{D}^{\prime}\right),
$$

where $n^{\prime}\left(x^{\prime}, t^{\prime}\right), F^{\prime}\left(x^{\prime}, t^{\prime}\right), J^{\prime}\left(x^{\prime}, t^{\prime}\right)$ are, respectively, the volume electron density, electric field, and current density, $x^{\prime}$ and $t^{\prime}$ are the spatial coordinate and time, $n_{D}^{\prime}$ is the $n$-type doping density in the SL layers, $e>0$ is the electron charge, and $\varepsilon_{0}$ and $\varepsilon_{r}$ are, respectively, the absolute and relative permittivities.

In analytical and numerical studies, it is more convenient to consider the dimensionless equations

$$
\begin{aligned}
\frac{\partial n}{\partial t} & =-\beta \frac{\partial J}{\partial x}, \\
\frac{\partial F}{\partial x} & =v(n-1),
\end{aligned}
$$

which can be obtained by introducing the following substitutions:

$$
\begin{aligned}
x & =x^{\prime} / L^{\prime}, \quad t=t^{\prime} / \tau^{\prime}, \quad n=n^{\prime} / n_{D}^{\prime}, \\
J & =J^{\prime} /\left(e n_{D}^{\prime} v_{0}^{\prime}\right), \quad F=F^{\prime} / F_{c}^{\prime}, \quad F_{c}^{\prime}=\hbar /\left(e d^{\prime} \tau^{\prime}\right), \\
\beta & =v_{0}^{\prime} \tau^{\prime} / L^{\prime}, \quad v=L^{\prime} e n_{D}^{\prime} /\left(F_{c}^{\prime} \varepsilon_{0} \varepsilon_{r}\right), \\
v_{0}^{\prime} & =\delta \Delta^{\prime} d^{\prime} /(2 \hbar) .
\end{aligned}
$$

Here, $n(x, t), F(x, t), J(x, t)$ are the dimensionless volume electron density, electric field, and current density, $x$ and $t$ the dimensionless coordinate and time, and $v$ and $\beta$ are dimensionless control parameters. In this paper, the values of the SL parameters in Eq. (5) are taken from recent experiments: ${ }^{32,43}$ $d^{\prime}=8.3 \mathrm{~nm}$ and $L^{\prime}=115.2 \mathrm{~nm}$ are the period and the length of the superlattice, $\Delta^{\prime}=19.1 \mathrm{meV}$ is the miniband width, and $F_{c}^{\prime}=3.1725 \times 10^{5} \mathrm{~V} / \mathrm{m}$ is the normalizing electric field. The parameters $\delta=\left[\tau_{e}^{\prime} /\left(\tau_{e}^{\prime}+\tau_{i}^{\prime}\right)\right]^{1 / 2}$ and $\tau^{\prime}=\delta \tau_{i}^{\prime}$ characterize the electron scattering and depend on the elastic $\tau_{e}^{\prime}$ and inelastic $\tau_{i}^{\prime}$ scattering times. In our study, we fix $\tau^{\prime}=250 \mathrm{fs}, \delta=1 / 8.5$, $n_{D}^{\prime}=3 \times 10^{22} \mathrm{~m}^{-3}$, and $\varepsilon_{r}=12.5$.

Within the drift-diffusion approximation, the current density is given by

$$
J=n v_{d}(F)-D(F) \frac{\partial n}{\partial x},
$$

where $v_{d}(F)$ is the dimensionless electron drift velocity, which relates to the dimensional drift velocity $v_{d}^{\prime}$, as $v_{d}=v_{d}^{\prime} / v_{0}^{\prime}$, and $D(F)$ is the diffusion coefficient, ${ }^{25}$ which can be neglected for low temperatures $T^{\prime}{ }^{6,25}$ Note that for the periodic boundary conditions, Eq. (6) can be derived directly from the Boltzmann transport equation. ${ }^{44}$

If we only apply an electric field $F$ to the SL, the drift velocity $v_{d}(F)$ can be expressed analytically by ${ }^{23}$

$$
v_{d}(F)=\frac{I_{1}\left[\Delta^{\prime} /\left(2 k_{B}^{\prime} T^{\prime}\right)\right]}{I_{0}\left[\Delta^{\prime} /\left(2 k_{B}^{\prime} T^{\prime}\right)\right]} \frac{F}{1+F^{2}},
$$

where $k_{B}$ is the Boltzmann constant and $I_{0,1}(x)$ are the modified Bessel functions of the first kind. However, if an additional magnetic field is applied to the SL, $v_{d}(F)$ should be obtained numerically, e.g., by the approach described in Refs. 45 and 46. by

The dimensionless voltage $V$ applied to the SL is defined

$$
V=U+\int_{0}^{1} F d x,
$$

where the (dimensionless) voltage drop across the contacts $U$ includes the effect of charge accumulation and depletion in the emitter and collector regions and a contact resistance. ${ }^{6,47}$ In order to find the dimensional values of applied voltage and the voltage dropped across contacts, one needs to multiply their dimensionless values by the normalizing factor $F_{c}^{\prime} L^{\prime}$.

We assume the Ohmic contacts (with a high electrical conductivity $\sigma^{\prime}=3788 \mathrm{Sm}^{-1}$ ), which set the boundary condition

$$
J(0, t)=s F(0, t)
$$


where $s=\sigma^{\prime} F_{c}^{\prime} /\left(e n_{D}^{\prime} v_{0}^{\prime}\right)=17.6511$ is the dimensionless control parameter corresponding to the electrical conductivity of the emitter contact.

For our numerical simulations, we use an explicit numerical scheme ${ }^{6,48}$ with $\Delta x=2.08 \times 10^{-3}$ and $\Delta t=5 \times 10^{-4}$. In order to avoid spurious current oscillations in the generation regimes $^{25}$ for the calculation of drift velocity, we use a value of the electric field averaged over $\approx 30$ layers (corresponding to one quantum well). ${ }^{6}$ The dimensional electrical current through the SL can therefore be obtained from

$$
I^{\prime}\left(t^{\prime}\right)=A^{\prime} e n_{D}^{\prime} v_{0}^{\prime} J\left(1, t^{\prime} / \tau^{\prime}\right)
$$

where $A^{\prime}=5 \times 10^{-10} \mathrm{~m}^{2}$ is the cross-sectional area of the superlattice. . $^{6,25,43}$

\section{GENERAL APPROACH TO CALCULATION OF LYAPUNOV EXPONENTS}

Although a continuous spatially extended system could be discretized in space and thus reduced to the set of ODEs, the result of the direct application of the standard procedures ${ }^{11,14}$ to the calculation of the spectrum of Lyapunov exponents depends on the step of discretization. ${ }^{17}$ Moreover, the physical meaning of such Lyapunov exponents is vague since their number and the corresponding Lyapunov vectors depend on the method of discretization. In addition, linearization of the discretized system is often either cumbersome, or not obvious. For example, in Ref. 28, where the dynamics of charge in a miniband SL subjected to an ac field was studied, the authors were unable to reliably calculate the Lyapunov exponents by the methods developed for ODEs, and therefore limited their Lyapunov stability analysis by the calculation of the largest exponents from the time series.

In our work, for the calculation of the spectrum of Lyapunov exponents from the set of Poisson and continuity equations we introduce perturbations, which are continuous both in time and in space. This approach allows us to avoid the problems associated with the artificial spatial discretization, provides one with clear interpretation of the results, and, in some cases, allows analytical estimation of the Lyapunov exponents as will be shown in the following.

It can be seen from Eqs. (3), (4), and (6) that the charge density $n(x, t)$ unambiguously defines all other variables of the model and, hence, the state of the system. Therefore, it is suitable for using as a reference state in the analysis of the evolution of small perturbations applied to asymptotic solutions of the system under study.

In order to compute the $N$ th largest Lyapunov exponents $\Lambda_{N}$, we consider a set of perturbations $\tilde{n}_{i}(x, t), i=1, \ldots, N$, which are initially orthogonal so that

$$
\left(\tilde{n}_{i}(x, 0), \tilde{n}_{j}(x, 0)\right)= \begin{cases}1, & i=j \\ 0, & i \neq j\end{cases}
$$

where $\left(\tilde{n}_{i}, \tilde{n}_{j}\right)$ is the scalar product

$$
\left(\tilde{n}_{i}, \tilde{n}_{j}\right)=\int_{0}^{1} \tilde{n}_{i} \tilde{n}_{j} d x .
$$

In addition to the orthogonality requirement defined by Eq. (11), $\tilde{n}_{i}(x, t)$ must also obey the normalization condition

$$
\left\|\tilde{n}_{i}\left(x, t_{r}\right)\right\|=1,
$$

where $\left\|\tilde{n}_{i}\right\|=\sqrt{\left(\tilde{n}_{i}, \tilde{n}_{i}\right)}$. We obtain the set of perturbations $\tilde{n}_{i}(x, t)$, which fulfill the requirements in Eqs. (11) and (12), when $t=t_{r}$ by applying the Gram-Schmidt procedure:

$$
\hat{n}_{1}\left(x, t_{r}\right)=\varphi_{1}(x),
$$

$$
\begin{gathered}
\hat{n}_{i+1}\left(x, t_{r}\right)=\varphi_{i+1}(x)-\sum_{k=1}^{i}\left[\tilde{n}_{k}\left(x, t_{r}\right), \varphi_{i+1}(x)\right] \tilde{n}_{k}\left(x, t_{r}\right), \\
i=1,2, \ldots, N-1 ; \quad \tilde{n}_{i}\left(x, t_{r}\right)=\frac{\hat{n}_{i}\left(x, t_{r}\right)}{\left\|\hat{n}_{i}\left(x, t_{r}\right)\right\|}, \\
i=1,2, \ldots, N .
\end{gathered}
$$

Here, $\varphi_{1}(x), \varphi_{2}(x), \ldots, \varphi_{N}(x)$ is a set of arbitrary, linearly independent functions, defined for the system under study.

To calculate the spectrum of Lyapunov exponents one has to follow the time evolution of both the reference asymptotic solution $n(x, t)$ and all its perturbations $\tilde{n}_{i}(x, t)$ $(i=1,2, \ldots, N)$. The dynamics of $n(x, t)$ is described by Eqs. (3), (4), (6), and (7) together with boundary conditions (8) and (9), whereas the evolution of the perturbations $\tilde{n}_{i}(x, t)$ in the vicinity of the reference state $n(x, t)$ are defined by the following linearized equations:

$$
\begin{aligned}
\frac{\partial \tilde{n}_{i}}{\partial t} & =-\beta \frac{\partial \tilde{J}_{i}}{\partial x}, \quad \frac{\partial \tilde{F}_{i}}{\partial x}=v \tilde{n}_{i}, \\
\tilde{J}_{i} & =\tilde{n}_{i} v_{d}(F)+n \frac{d v_{d}(F)}{d F} \tilde{F}_{i}, \\
\tilde{U}_{i}+\int_{0}^{1} \tilde{F}_{i} d x & =0, \quad \tilde{J}_{i}(0, t)=s \tilde{F}_{i}(0, t) .
\end{aligned}
$$

Finite precision of numerical calculations requires repetition of the Gram-Schmidt procedure after some reasonable time $\Gamma$ (Ref. 11) with newly defined functions

$$
\varphi_{i}(x)=\tilde{n}_{i}(x, \Gamma) .
$$

The above algorithm should be repeated many times. After $M$ iterations, the perturbations $\tilde{n}_{i}\left(x, t_{r}\right)$, at $t_{r}=j \Gamma$ $(j=1, \ldots, M)$ obtained after orthogonalization [Eqs. (13) and (14)], but before renormalization [Eq. (16)], are used to calculate the Lyapunov sums

$$
S_{i}=\sum_{j=1}^{M} \ln \left\|\tilde{n}_{i}(x, j \Gamma)\right\| .
$$

Finally, the Lyapunov exponents are estimated as

$$
\Lambda_{i}=\frac{S_{i}}{M \Gamma}
$$

The Lyapunov exponents estimated by formula (19) have clear physical meaning. Namely, they describe the increment of growth (damping decrement) of the orthogonal modes of the perturbations.

The same approach can be used for the calculation of the Lyapunov exponents for a nonautonomous model of a SL, for example, when the SL is subjected to an ac voltage

$$
V(t)=V_{0}+\Delta V_{0} \cos \left(\omega_{e} t\right)
$$


where $V_{0}$ is the dc component of the applied voltage, and $\Delta V_{0}$ and $\omega_{e}$ are, respectively, the amplitude and angular frequency. However, in this case, the above algorithm returns the so-called conditional Lyapunov exponents, ${ }^{49}$ which only characterize the convergence behavior of the driven subsystem, and are therefore unable to reveal an additional zeroth Lyapunov exponent corresponding to the harmonic external force.

\section{ANALYTICAL ESTIMATION OF LYAPUNOV EXPONENTS FOR STEADY-STATE SOLUTIONS}

In general, the Lyapunov exponents for the above SL model can only be calculated numerically. However, in the case of an applied dc voltage in a steady-state regime, the Lyapunov exponents can be estimated analytically.

For the steady-state solution, $\partial n / \partial t=0, \partial F / \partial t=0$, and, consequently,

$$
n=n(x), \quad F=F(x), \quad n v_{d}(F)=J_{0}=\text { const. }
$$

Although the transport region of SL is defined in the interval $x \in[0,1]$, the electric field $F(x)$ can be formally introduced for any $x \in[0, \infty]$. Therefore, we denote

$$
f=\lim _{x \rightarrow \infty} F(x)
$$

and perform a Taylor expansion of $v_{d}(F)$ [Eq. (7)] around the point $F=f$ :

$$
\begin{aligned}
v_{d}(F) & \approx a_{0}+a_{1}(F-f)+O\left[(F-f)^{2}\right], \\
a_{0} & =\frac{f}{1+f^{2}}, \quad a_{1}=\frac{1-f^{2}}{\left(1+f^{2}\right)^{2}} .
\end{aligned}
$$

Taking into account Eqs. (21)-(23), we can now approximate Eq. (4) as

$$
\frac{d F}{d x} \approx v\left(\frac{J_{0}}{a_{0}+a_{1}(F-f)}-1\right),
$$

which has the solution

$$
\begin{aligned}
F(x)= & \frac{1}{a_{1}}\left\{a_{1} f+J_{0}-a_{0}\right. \\
& \left.+J_{0} W\left[\frac{1}{J_{0}} \exp \left(-1+\frac{a_{0}-a_{1}(f+v x)+a_{1} C}{J_{0}}\right)\right]\right\},
\end{aligned}
$$

where $W(z)$ is the Lambert $W$ function, and $C$ is an integration constant. The value of $J_{0}$ can be found using Eq. (22):

$$
\lim _{x \rightarrow \infty} F(x)=f+\frac{J_{0}-a_{0}}{a_{1}}=f,
$$

which gives $J_{0}=a_{0}$, and $C$ is determined by the boundary condition in Eq. (9):

$$
C=\frac{a_{0}}{s}+\frac{a_{0}}{a_{1}} \ln \left(\frac{a_{1}\left(a_{0}-s f\right)}{s}\right) .
$$

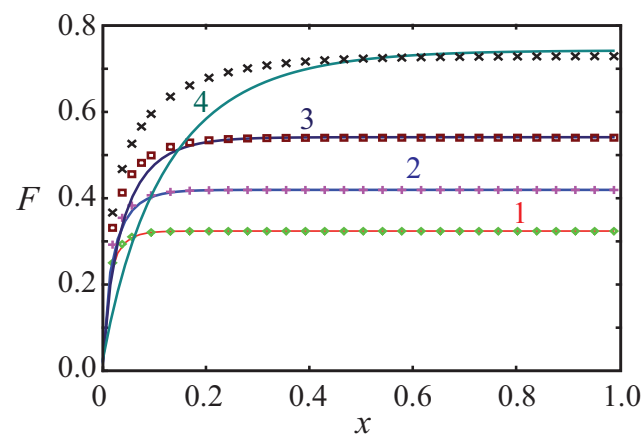

FIG. 1. (Color online) The dependencies $F(x)$ for various $V$, calculated both analytically using Eq. (28) (solid curves) and numerically (symbols). Curve $1(\diamond)$ corresponds to $V=4.9252$ $\left(V^{\prime}=180 \mathrm{mV}\right)$; curve $2(+), V=6.0197\left(V^{\prime}=220 \mathrm{mV}\right)$; curve $3(\boldsymbol{\square}), V=7.1142\left(V^{\prime}=260 \mathrm{mV}\right)$; and curve $4(\times), V=8.2087$ $\left(V^{\prime}=300 \mathrm{mV}\right)$.

After substitution of the expressions for $J_{0}, C, a_{0}$, and $a_{1}$ into (25) we obtain

$$
\begin{aligned}
F(x)= & f+f \frac{1+f^{2}}{1-f^{2}} W\left[\frac{\left(f^{2}-1\right)\left(s-1+s f^{2}\right)}{\left(f^{2}+1\right)^{2} s}\right. \\
& \left.\times \exp \left(\frac{\left(f^{2}-1\right)\left[s v x+s f^{3}+s v x f^{2}+f(s-1)\right]}{\left(f^{2}+1\right)^{2} s f}\right)\right],
\end{aligned}
$$

where the value of $f$ can be found from the voltage constraint (8).

We compare $F(x)$ obtained using Eq. (28) (solid curve in Fig. 1) and $F(x)$ calculated numerically (symbols in Fig. 1) for a range of $V$. Figure 1 shows that for low voltages $V<$ $7.1142\left(V^{\prime}<260 \mathrm{mV}\right)$, Eq. (28) is in excellent agreement with numerical simulations. However, as $V$ approaches the threshold voltage $V_{t h}$, where $I(t)$ loses its stability and starts to demonstrate high-frequency oscillations, the estimation (28) starts to deviate from numerical calculations because the Taylor series expansion of drift velocity [Eq. (23)] becomes less accurate. Figure 1 shows that for a range of $V$, the electric field $F(x)$ is almost spatially homogeneous, except for a small region of $x$ near the emitter. Therefore, we can use the approximation

$$
F(x) \simeq \text { constant }=f,
$$

which, according to Eq. (4), yields $n(x)=1$. By rearranging Eqs. (16), we obtain a linearized equation for the perturbations of the stationary state

$$
\frac{\partial \tilde{n}}{\partial t}=-v_{d}(f) \beta \frac{\partial \tilde{n}}{\partial x}-\frac{d v_{d}(f)}{d F} \beta v \tilde{n} .
$$

We search for a solution in the form of plane wave $\tilde{n} \sim$ $\exp [j(\omega t+k x)](j=\sqrt{-1})$, which leads to the dispersion relation

$$
\omega=-v_{d}(f) \beta k+j \frac{d v_{d}(f)}{d F} \beta v,
$$

which is used for evaluation of the Lyapunov exponents $\Lambda_{t}$ :

$$
\Lambda_{t}=-\frac{\ln \|\tilde{n}(x, t)\|}{t}=-\frac{d v_{d}(f)}{d F} \beta \nu .
$$




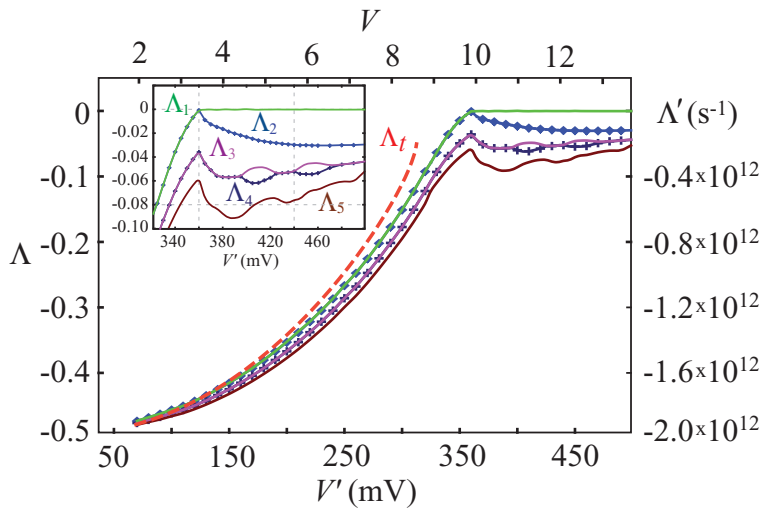

FIG. 2. (Color online) The five largest Lyapunov exponents $\left(\Lambda_{1}-\Lambda_{5}\right)$ versus voltage $V$ for a dc-biased SL. The symbols, joined by a solid curve, show the numerically calculated exponents, whereas the analytical result [Eq. (32)] is represented by the dashed curve. The lower and right-hand axes (upper and left-hand axes) are in SI (dimensionless) units. Inset shows an enlarged region of $V$ near $V_{t h}$.

Equation (32) indicates that within the regimes outlined above, any perturbation is characterized by the same Lyapunov exponent. This means that all orthogonal perturbations of the steady state have the same growth rate (instability increment). Moreover, according to Eq. (32), in the case of homogeneous field (29), the Lyapunov exponent has a meaning of the dielectric relaxation rate $^{25}$ taken with opposite sign. In addition, it follows from Eq. (32) that the steady state loses its stability at the critical value of electric field

$$
f_{c}=1,
$$

where $\Lambda_{t}$ changes sign.

Figure 2 shows that for low voltages, the five largest Lyapunov exponents $\left(\Lambda_{1}-\Lambda_{5}\right)$ versus $V$ calculated numerically (symbols joined by solid curve in Fig. 2) agree well with the analytic estimation given by Eq. (32) (dashed line labeled $\Lambda_{t}$ in Fig. 2). However, as $V$ increases the analytical estimation becomes less precise since, as Fig. 1 shows, for large $V$ the approximation given in Eq. (29) becomes invalid. Note as $L^{\prime}$ increases, the range of $x$, where $F(x) \approx$ const, also

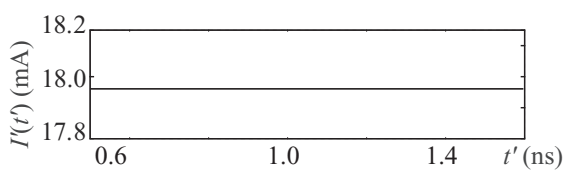

(a)
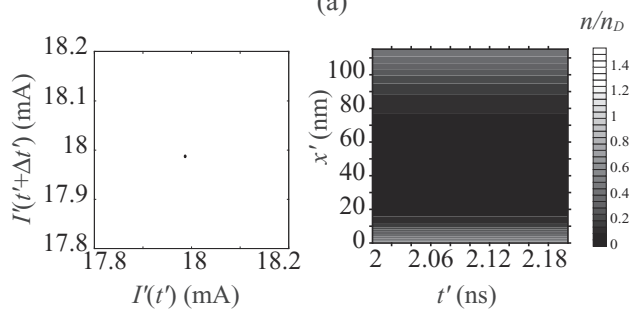

(b)

(c)

grows. Thus, the analytically estimated Lyapunov exponents are more accurate for longer SLs.

We compare Eq. (33) with the conventional $n L$ criterion, ${ }^{36}$ which is traditionally used as a condition for the onset of charge-domain instability in Gunn diodes ${ }^{50}$ and $\mathrm{SLs}^{51}$ and can be expressed by

$$
n^{\prime} L^{\prime}>2.09 \frac{\varepsilon_{0} \varepsilon_{r}}{e} \frac{F^{\prime 2}+F_{c}^{\prime 2}}{F^{\prime 2}-F_{c}^{\prime 2}} F^{\prime} .
$$

In the dimensionless form, the above Eq. (34) reads as

$$
n>\alpha \frac{F^{2}+1}{F^{2}-1} F,
$$

where $\alpha=2.09 v^{-1}$. Rearranging Eq. (35) and taking into account Eq. (7), we can find the critical value of $F$ corresponding to the $n L$ criterion

$$
F_{n L}=\sqrt{\frac{J}{J-\alpha}} .
$$

For long SLs, $\alpha \rightarrow 0$, and the $n L$ criterion coincides with the condition (33).

\section{DIFFERENT DYNAMICAL REGIMES AND THEIR STABILITY}

The numerically calculated Lyapunov exponents presented in Fig. 2 predict that the steady state of the SL, with parameters given in Sec. II, loses its stability at the critical voltage $V_{t h}=9.85\left(V_{t h}^{\prime}=360 \mathrm{mV}\right) .^{52}$ To understand the physics behind this instability we consider the numerical simulations of the spatiotemporal charge dynamics in the SL.

Figure 3 shows the electron transport dynamics of two characteristic regimes. When $V=9.71347\left(V^{\prime}=355 \mathrm{mV}\right), I(t)$ does not oscillate [Fig. 3(a)], corresponding to a fixed point in phase space (constructed by the delay-coordinate embedding method $^{53}$ with time delay $\Delta t^{\prime}=10$ ps) [see Fig. 3(b)]. In this case, $n(x, t)$ is time independent and is distributed almost uniformly through the SL, as shown in Fig. 3(c). However, by making a small change of $V$ to $9.98709\left(V^{\prime}=365 \mathrm{mV}\right)$, the behavior of the charge dramatically changes. We find that at this voltage, $I(t)$ performs periodic oscillations [Fig. 3(d)],

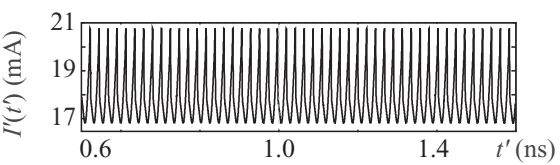

(d)

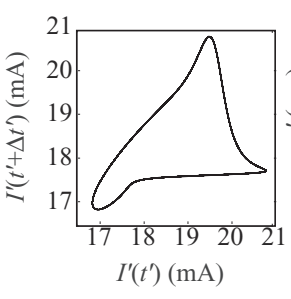

(e)

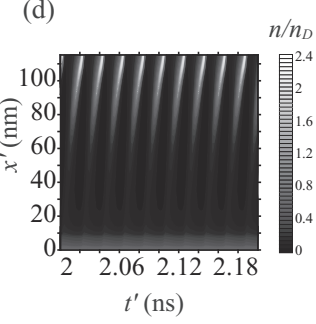

(f)

FIG. 3. The steady-state (a)-(c) and time-periodic (d)-(f) regimes. The steady state: (a) $I(t)$; (b) projection of the attractor onto the reconstructed phase space; and (c) surface plot of $n(x, t)$. The time-periodic regime: (d) $I(t)$; (e) projection of the attractor onto the reconstructed phase space $\left[I^{\prime}\left(t^{\prime}\right), I^{\prime}\left(t+\Delta t^{\prime}\right)\right]$ with $\Delta t^{\prime}=10 \mathrm{ps}$; and (f) surface plot of $n(x, t)$. 

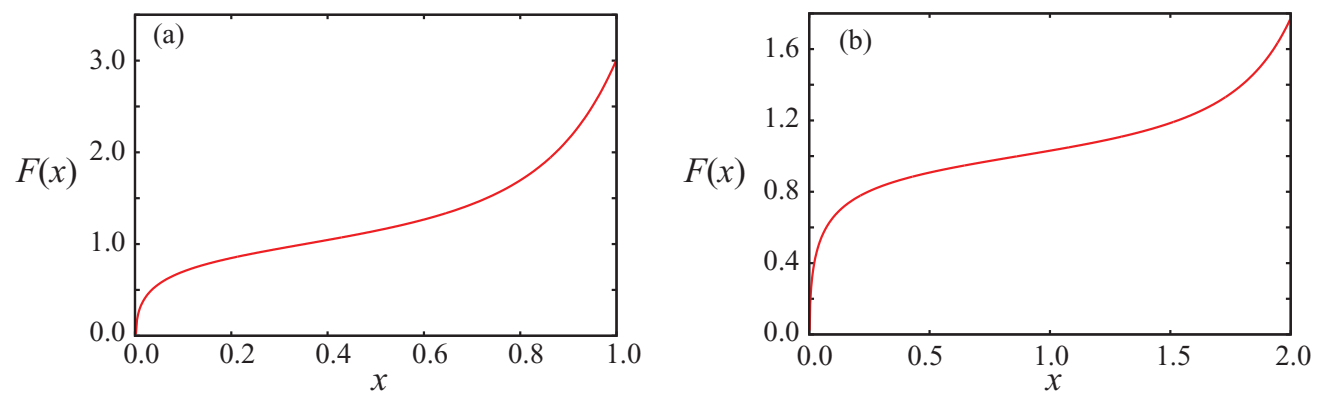

FIG. 4. (Color online) The dependencies of the electric field $F$ upon the space coordinate $x$ for two values of the length of the SL (in the dimensionless variables): (a) $L=1$ and (b) $L=2$. Both curves correspond to the voltages, which are very close to the critical values.

which are represented by a limit cycle in the phase space [Fig. 3(e)]. Figure 3(f) shows that these current oscillations are caused by domains of high-charge concentration. A domain is induced at the emitter $(x=0)$ and then propagates through the SL. When the domain reaches the collector, a new domain is induced at the emitter and the cycle repeats. ${ }^{6}$ These domains are only observed after the steady-state solution loses its stability. Thus, the Lyapunov exponents give a reliable way to distinguish between steady-state and periodic dynamics, and to identify the bifurcation transition between these regimes.

Note that the $n L$ criterion (34) underestimates the critical voltage of these SL parameters, giving $V_{t h}^{\prime} \approx 328.7 \mathrm{mV}$ (compared with the numerical obtained value of $V_{t h}^{\prime} \approx 360 \mathrm{mV}$ ). The reason for this discrepancy is that the condition in Eq. (34) was derived assuming a homogeneous electric field. ${ }^{36}$ In our SL, as the voltage approaches the critical value, the electric field through the SL becomes less homogeneous, especially in the emitter and collector regions. In addition, the voltage drop across the SL's contacts can also influence $V_{t h}^{\prime}$. However, we note that the $n L$ criterion becomes more accurate for a longer SL. For example, for the SL with the same parameters, but with a length of $230.4 \mathrm{~nm}(L=2)$, the $n L$ criterion gives $V_{t h}^{\prime} \approx 375.5 \mathrm{mV}$, which is very close to the numerically estimated value of $378.3 \mathrm{mV}$. Remarkably, doubling of the SL's length $L$ leads to only small increase of the critical voltage $V_{t h}^{\prime}$. This could be explained again by inhomogeneous electric field distribution $F(x)$ across the SL, which is especially pronounced near the contacts. To illustrate this, in Fig. 4 we plot the dependencies $F(x)$ for two values of the length of the SL (in the dimensionless variables) for the voltages very close to $V_{t h}$. Namely, in Fig. 4(a), $F(x)$ is presented for $L=1$ and $V=9.823\left(V^{\prime}=359 \mathrm{mV}\right)$, and Fig. 4(b) represents $F(x)$ for $L=2$ and $V=10.343\left(V^{\prime}=378 \mathrm{mV}\right)$. One can see that the magnitude of $F$ corresponding to the critical voltage considerably decreases with the growth of the length of the SL. As a consequence, the integral $\int_{0}^{L} F(x) d x$ in Eq. (8), defining the voltage drop across transport area of the SL, increases only slightly. At the same time, in both cases $F(x)$ far from the contacts varies slowly and demonstrates values close to one, which agrees with the criterion (33) derived within the homogeneous field approximation.

In order to examine the behavior of the Lyapunov exponents in other dynamical regimes, we consider a nonautonomous SL under the influence of a tilted static magnetic field $\mathbf{B}=(B \cos \theta, 0, B \sin \theta)$ and ac voltage in the form given by Eq. (20). The effect of magnetic field is taken into account through the calculations of $v_{d}(F)$, using the approach described in detail in Refs. 43, 45, and 46. In our numerical simulations, we fix $B=12 \mathrm{~T}$ and $\theta=40^{\circ}$.

Three different dynamical regimes are shown in Fig. 5. When the ac voltage parameters are $V_{0}=16.691$, $\Delta V_{0}=2.736$, and $\omega_{e}=3.299 \times 10^{-2} \quad\left(V_{0}^{\prime}=610 \mathrm{mV}\right.$, $\Delta V_{0}^{\prime}=100 \mathrm{mV}$, and $\left.f_{e}^{\prime}=\omega_{e}^{\prime} / 2 \pi=21.0 \mathrm{GHz}\right), I(t)$ oscillates periodically [Fig. 5(a)], and is represented in the phase space by a limit cycle [Fig. 5(b)]. The periodic oscillations of $I(t)$ reflect a periodic spatiotemporal pattern in the dynamics of $n(x, t)$ [see Fig. 5(c)]. The character of $I(t)$ dramatically changes if the frequency of the external force is only slightly changed. If the frequency is increased up to $\omega_{e}=3.691 \times 10^{-2}\left(f_{e}^{\prime}=23.5 \mathrm{GHz}\right)$ we find that the the amplitude of the current oscillations starts to vary periodically [see Fig. 5(d)], which corresponds to quasiperiodic dynamics and results in a ergodic torus in the phase space of the system [Fig. 5(e)]. The form of $n(x, t)$ is shown in Fig. 5(f), where the quasiperiodic character of the dynamics is reflected in the modulation of the speed that the charge domain travels from the emitter to the collector.

By increasing the frequency of the ac voltage further, for example, setting $\omega_{e}=2.906 \times 10^{-2}\left(f_{e}^{\prime}=18.5 \mathrm{GHz}\right)$, we find the oscillations of $I(t)$ become irregular [Fig. 5(g)]. This is confirmed in Fig. 5(h), where a projection of the phase trajectories onto the plane $\left[I^{\prime}\left(t^{\prime}\right), I\left(t^{\prime}+\Delta t^{\prime}\right)\right]$ suggests chaotic behavior. In this case, the motion of charge domains becomes very erratic [see Fig. 5(i)], different charge domains appear and disappear irregularly and propagate with different speeds. These observations suggest that, in this regime, the electron transport in the SL is attributed to deterministic chaos.

We note that the interesting dynamical regimes described above can be associated with synchronization and desynchronization of periodic oscillations ${ }^{54}$ related to the charge transport in SL, and deserve further study. However, in this paper we only use these characteristic regimes to test the proposed method for calculation of the Laypunov exponents.

In Fig. 6, we show the highest conditional Lyapunov exponent $\Lambda_{1}$ versus $t^{\prime}$, calculated for the three characteristic dynamical regimes discussed above and illustrated in Fig. 5. In order to investigate the convergence of the exponents, we analyze their dependence on time using Eq. (19), where $t^{\prime}=M \Gamma^{\prime}$, for $M=1,2, \ldots$ and $\Gamma^{\prime}=6.25$ ps.

For the periodic solution illustrated in Figs. 5(a)-5(c), $\Lambda_{1}$ is always negative (curve 1 in Fig. 6). In the quasiperiodic regime shown in Figs. 5(d)-5(f), $\Lambda_{1}$ is close to zero (curve 2 


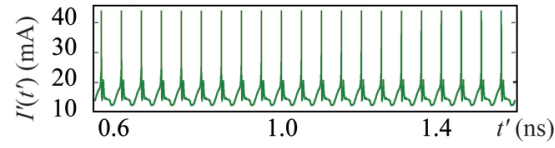

(a)

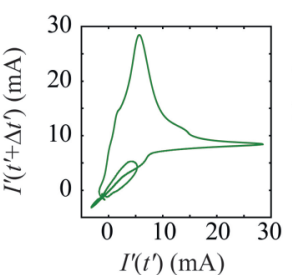

(b)

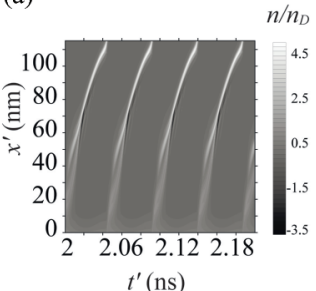

(c)

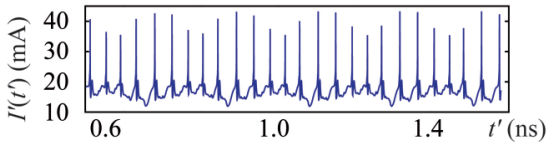

(d)

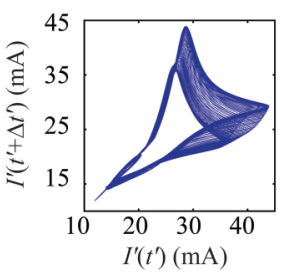

(e)

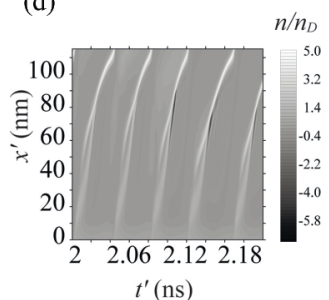

(f)

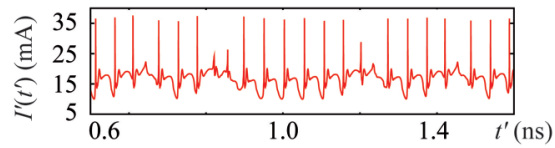

(g)

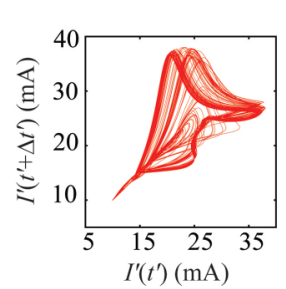

(h)

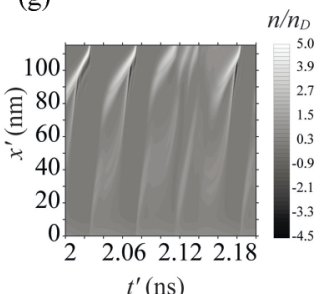

(i)

FIG. 5. (Color online) Periodic (a)-(c), quasiperiodic (d)-(f), and chaotic (g)-(i) dynamics of charge in SL: oscillations of the current $I(t)$ $[(\mathrm{a}),(\mathrm{d}),(\mathrm{g})]$; projection of the attractor onto the plane $\left[I^{\prime}\left(t^{\prime}\right), I^{\prime}\left(t+\Delta t^{\prime}\right)\right], \Delta t^{\prime}=10 \mathrm{ps}$, in the reconstructed phase space [(b), (e), (h)]; and spatiotemporal dynamics of the volume electron density $n(x, t)[(\mathrm{c}),(\mathrm{f}),(\mathrm{i})]$.

in Fig. 6). Finally, for the parameters corresponding to the dynamical regime in Figs. $5(\mathrm{~g})-5(\mathrm{i}), \Lambda_{1}$ is positive for all $t^{\prime}$, which indicates the Lyapunov instability and confirms the chaotic character of the dynamics. Note, as discussed in Sec. III, the conditional Lyapunov exponents do not take into account the zeroth Lyapunov exponent corresponding to the phase change of the external driving, therefore the largest conditional Lyapunov exponent corresponding to periodic dynamics (curve 1 in Fig. 6) is negative. Figure 6 also shows that the convergence of $\Lambda_{1}\left(t^{\prime}\right)$ strongly depends on the dynamical regime. In the periodic and quasiperiodic regimes, the spatiotemporal patterns of $n(x, t)$ need $\approx 20 \mathrm{~ns}$ before $\Lambda_{1}\left(t^{\prime}\right)$ converges to a stationary value. In contrast, the

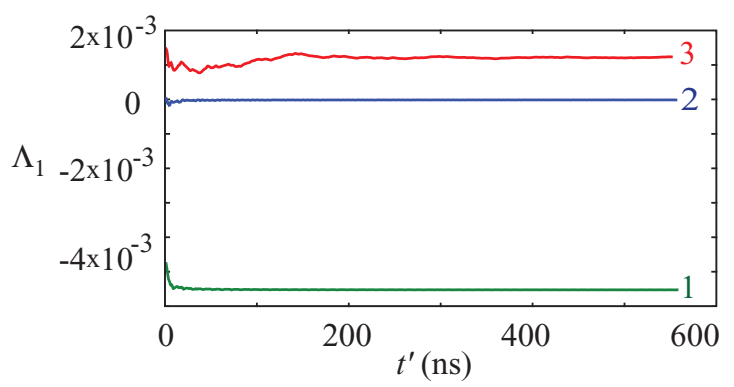

FIG. 6. (Color online) $\Lambda_{1}$ versus $t^{\prime}$ obtained for synchronous periodic (curve 1), quasiperiodic (curve 2), and chaotic (curve 3) dynamical regimes. chaotic dynamics requires a longer time $(\approx 200 \mathrm{~ns})$ before convergence.

To illustrate the transitions between the regimes discussed, in Fig. 7, we show how the five highest conditional Lyapunov exponents $\left(\Lambda_{1}-\Lambda_{5}\right)$ vary with ac voltage frequency $\left(f_{e}^{\prime}\right)$. The SL parameter values have been selected to be the same as those in Fig. 5 with $f_{e}^{\prime}$ corresponding to the different regimes indicated by numbered arrows. We find that the three characteristic regimes discussed previously are robust against change in parameter. Specifically, for $f_{e}^{\prime}$ between $\approx 18$ and

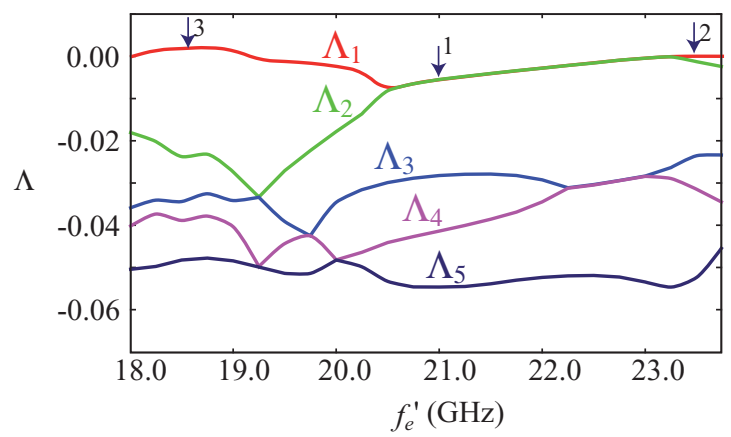

FIG. 7. (Color online) The five highest conditional Lyapunov exponents $\left(\Lambda_{1}-\Lambda_{5}\right)$ versus ac voltage frequency $f_{e}^{\prime}$. The frequencies corresponding to the characteristic regimes in Fig. 5 are indicated by arrows, where 1 corresponds to the periodic regime $\left(\Lambda_{1}<0\right), 2$ to the quasiperiodic regime $\left(\Lambda_{1} \approx 0\right)$, and 3 to the chaotic regime $\left(\Lambda_{1}>0\right)$. 
$\approx 19.2 \mathrm{GHz}$, the electron transport in SL demonstrates chaotic dynamics, characterized by $\Lambda_{1}>0$. For $f_{e}^{\prime}$ between $\approx 19.2$ and $23 \mathrm{GHz}, \Lambda_{1}<0$ indicating periodic behavior. Finally, for $f_{e}^{\prime}>$ $23 \mathrm{GHz}$ the transport dynamics is quasiperiodic, and $\Lambda_{1}=0$.

\section{CONCLUSIONS}

We have proposed a method for numerical calculation of the Lyapunov exponents for a set of one-dimensional Poisson and continuity equations. The method is based on the analysis of orthogonal perturbations applied to a stationary solution of charge density. The proposed approach has been applied both to an autonomous and to a nonautonomous miniband SL subjected to an ac voltage and tilted magnetic field.

In the case that a dc voltage is applied to the SL, the Lyapunov exponents of the steady-state solution can be estimated analytically. If the electric field is uniform along the SL, all the perturbations are characterized by the same Lyapunov exponent, which is also confirmed by our numerical calculations. The analytically calculated Lyapunov exponents can be used for an estimation of the critical voltage, at which the steady state loses its stability, and $I(t)$ starts to oscillate. We show that for long SLs this critical voltage agrees well with that obtained from the $n L$ criterion, ${ }^{36,50}$ traditionally used in the Gunn diodes and SLs to determine their stability properties.

When the SL is subjected to an ac voltage and a tilted magnetic field, the proposed technique is able to unambiguously reveal periodic, quasiperiodic, and chaotic collective electron dynamics. Without ac voltage our approach applied to a realistic model of a miniband SL shows a good agreement with the experimental measurements ${ }^{32}$ of the current oscillations threshold. Further analysis predicts that application of an ac field to a SL subjected to a tilted magnetic field can induce quiasiperiodic and even chaotic current oscillations. This finding suggests a way to create tunable high-frequency chaos generators based on SL structures.

In addition, another important class of problems which our method could be applied to is the electric stability of several, recently suggested, strongly nonlinear SL-based high-frequency amplifiers, including those pumped with microwaves, ${ }^{55,56}$ with an applied magnetic field, ${ }^{34}$ or operating in a large-signal regime. ${ }^{57,58}$ In these cases, application of the conventional $n L$ criterion $^{36}$ is limited, and Lyapunov exponents could give more accurate and reliable prediction of instabilities developed in the systems. Moreover, Lyapunov stability analysis can shed light on how transitions to various spatiotemporal dynamical regimes affect amplification in the above systems.

We believe that our method has also a great potential for analysis of the spatiotemporal dynamics of charge in Gunn diodes ${ }^{59-61}$ and plasma devices. ${ }^{2,3}$ For example, the knowledge of several largest Lyapunov exponents can be used for chaos control through "unstable periodic orbit stabilization" $3,4,62,63$ or for characterization of different types of chaos synchronization ${ }^{64-66}$ in plasma or electronic devices.

\section{ACKNOWLEDGMENTS}

This work has been supported by the Ministry of Education and Science of Russian Federation (Projects No. 14.B37.21.0059 and No. 14.B37.21.1207), President Programm (Projects No. MD-345.2013.2 and No. MK672.2012.2), Russian Foundation for Basic Research (Project No. 12-02-33071), and the "Dynasty" Foundation.
${ }^{1}$ H. Matsumoto, H. Yokoyama, and D. Summers, Phys. Plasmas 3, 177 (1996).

${ }^{2}$ J. M. Finn, D. del-Castillo-Negrete, and D. C. Barnes, Phys. Rev. Lett. 84, 2401 (2000).

${ }^{3}$ T. Klinger, C. Schroder, D. Block, F. Greiner, A. Piel, G. Bonhomme, and V. Naulin, Phys. Plasmas 8, 1961 (2001).

${ }^{4}$ A. E. Hramov, A. A. Koronovskii, and I. S. Rempen, Chaos 16, 013123 (2006).

${ }^{5}$ A. Amann, J. Schlesner, A. Wacker, and E. Schöll, Phys. Rev. B 65, 193313 (2002).

${ }^{6}$ M. T. Greenaway, A. G. Balanov, E. Schöll, and T. M. Fromhold, Phys. Rev. B 80, 205318 (2009).

${ }^{7}$ J. J. Brondijk, M. Spijkman, F. van Seijen, P. W. M. Blom, and D. M. de Leeuw, Phys. Rev. B 85, 165310 (2012).

${ }^{8}$ B. B. Godfrey, Phys. Fluids 30, 1553 (1987).

${ }^{9}$ T. Klinger, A. Latten, A. Piel, E. Bonhomme, and T. Pierre, Plasma Phys. Controlled Fusion 39, B145 (1997).

${ }^{10}$ A. Madon and T. Klinger, Phys. D (Amsterdam) 102, 335 (1997).

${ }^{11}$ A. Wolf, J. Swift, H. L. Swinney, and J. Vastano, Phys. D (Amsterdam) 16, 285 (1985).

${ }^{12}$ V. I. Oseledec, Trans. Moscow Math. Soc. 19, 197 (1968).

${ }^{13}$ G. Benettin, L. Galgani, and J. M. Strelcyn, Phys. Rev. A 14, 2338 (1976).
${ }^{14}$ G. Benettin, L. Galgani, A. Giorgilli, and J. M. Strelcyn, Meccanica 15, 9 (1980).

${ }^{15}$ S. Lepri, A. Politi, and A. Torcini, Chaos 7, 701 (1997).

${ }^{16}$ H. L. Yang and G. Radons, Philos. Trans. R. Soc., A 367, 3197 (2009).

${ }^{17}$ R. Carretero-Gonzalez, S. Orstavik, J. Huke, D. S. Broomhead, and J. Stark, Chaos 9, 466 (1999).

${ }^{18}$ A. S. Pikovsky and A. Politi, Nonlinearity 11, 1049 (1998).

${ }^{19}$ R. A. Filatov, A. E. Hramov, and A. A. Koronovskii, Phys. Lett. A 358, 301 (2006).

${ }^{20}$ A. E. Hramov, A. A. Koronovskii, and P. V. Popov, Phys. Rev. E 77, 036215 (2008).

${ }^{21}$ A. A. Koronovskii, P. V. Popov, and A. E. Hramov, J. Exp. Theor. Phys. 103, 654 (2006).

${ }^{22}$ S. P. Kuznetsov and D. I. Trubetskov, Radiophys. Quantum Electron. 47, 341 (2004).

${ }^{23}$ L. Esaki and R. Tsu, IBM J. Res. Dev. 14, 61 (1970).

${ }^{24}$ A. Y. Shik, Fiz. Tekh. Poluprovodn. 8, 1841 (1974) [Sov. Phys.Semicond. 8, 1195 (1975)].

${ }^{25}$ A. Wacker, Phys. Rep. 357, 1 (2002).

${ }^{26}$ O. M. Bulashenko and L. L. Bonilla, Phys. Rev. B 52, 7849 (1995).

${ }^{27}$ O. M. Bulashenko, M. J. Garcia, and L. L. Bonilla, Phys. Rev. B 53, 10008 (1996).

${ }^{28}$ J. C. Cao and X. L. Lei, Phys. Rev. B 60, 1871 (1999). 
${ }^{29}$ H. Eisele, S. Khanna, and E. Linfield, Appl. Phys. Lett. 96, 072101 (2010).

${ }^{30}$ C. P. Endres, F. Lewen, T. F. Giesen, S. Schlemmer, D. G. Paveliev, Y. I. Koschurinov, V. M. Ustinov, and A. E. Zhucov, Rev. Sci. Instrum. 78, 043106 (2007).

${ }^{31}$ D. G. Paveliev, Y. I. Koschurinov, A. S. Ivanov, A. N. Panin, V. L. Vax, V. I. Gavrilenko, A. V. Antonov, V. M. Ustinov, and A. E. Zhukov, Semiconductors 46, 121 (2012).

${ }^{32}$ N. Alexeeva, M. T. Greenaway, A. G. Balanov, O. Makarovsky, A. Patane, M. B. Gaifullin, F. Kusmartsev, and T. M. Fromhold, Phys. Rev. Lett. 109, 024102 (2012).

${ }^{33}$ K. Hofbeck, J. Grenzer, E. Schomburg, A. A. Ignatov, K. F. Renk, D. G. Paveliev, Y. I. Koschurinov, B. Melzer, S. Ivanov, S. Schaposchnikov et al., Phys. Lett. A 218, 349 (1996).

${ }^{34}$ T. Hyart, J. Mattas, and K. N. Alekseev, Phys. Rev. Lett. 103, 117401 (2009).

${ }^{35}$ J. Isohätälä and K. N. Alekseev, J. Phys.: Condens. Matter 24, 145303 (2012).

${ }^{36}$ D. E. McCumber and A. G. Chynoweth, IEEE Trans. Electron Devices ED-13, 4 (1966).

${ }^{37}$ R. Tsu, Superlattices to Nanoelectronics (Elsevier, Amsterdam, 2005).

${ }^{38}$ A. Gogolin, Zh. Eksp. Teor. Fiz. 88, 2063 (1985) [Sov. Phys.-JETP 61, 1220 (1985)].

${ }^{39}$ L. Person, C. Minot, L. Boni, J. F. Palmier, and F. Mollot, Appl. Phys. Lett. 60, 2397 (1992).

${ }^{40}$ A. A. Ignatov and V. I. Shashkin, Fiz. Tekh. Poluprovodn. 18, 721 (1984) [Sov. Phys. Semicond. 18, 449 (1984)].

${ }^{41}$ L. L. Bonilla, R. Escobedo, and A. Perales, Phys. Rev. B 68, 241304 (2003).

${ }^{42}$ J. Hizanidis, A. G. Balanov, A. Amann, and E. Schöll, Phys. Rev. Lett. 96, 244104 (2006).

${ }^{43}$ T. M. Fromhold, A. Patane, S. Bujkiewicz, P. B. Wilkinson, D. Fowler, D. Sherwood, S. P. Stapleton, A. A. Krokhin, L. Eaves, M. Henini et al., Nature (London) 428, 726 (2004).

${ }^{44}$ A. A. Ignatov and V. I. Shashkin, Zh. Eksp. Teor. Fiz. 93, 935 (1987) [Sov. Phys.-JETP 66, 526 (1987)].

${ }^{45}$ T. M. Fromhold, A. A. Krokhin, C. R. Tench, S. Bujkiewicz, P. B. Wilkinson, F. W. Sheard, and L. Eaves, Phys. Rev. Lett. 87, 046803 (2001).
${ }^{46}$ A. O. Selskii, A. A. Koronovskii, A. E. Hramov, O. I. Moskalenko, K. N. Alekseev, M. T. Greenaway, F. Wang, T. M. Fromhold, A. V. Shorokhov, N. N. Khvastunov et al., Phys. Rev. B 84, 235311 (2011).

${ }^{47}$ D. Hardwick, Ph.D. thesis, University of Nottingham, 2007.

${ }^{48}$ M. T. Greenaway, A. G. Balanov, D. Fowler, A. J. Kent, and T. M. Fromhold, Phys. Rev. B 81, 235313 (2010).

${ }^{49}$ L. M. Pecora and T. L. Carroll, Phys. Rev. A 44, 2374 (1991).

${ }^{50}$ H. Kroemer, IEEE Trans. Electron Devices ED-15, 819 (1968).

${ }^{51}$ N. V. Demarina, A. Lisauskas, and H. G. Roskos, Microelectron. J. 39, 624 (2008)

${ }^{52}$ This value of the critical voltage is in a very good agreement with recent experiments (Ref. 32) involving the SLs, whose parameters we used in our simulations.

${ }^{53} \mathrm{~F}$. Takens, in Lectures Notes in Mathematics, edited by D. Rand and L. S. Young (Springer, New York, 1981), p. 366.

${ }^{54}$ A. G. Balanov, N. B. Janson, D. E. Postnov, and O. V. Sosnovtseva, Synchronization: From Simple to Complex (Springer, Berlin, 2009).

${ }^{55}$ T. Hyart, N. Alexeeva, A. Leppanen, and K. N. Alekseev, Appl. Phys. Lett. 89, 132105 (2006).

${ }^{56}$ T. Hyart, N. V. Alexeeva, J. Mattas, and K. N. Alekseev, Phys. Rev. Lett. 102, 140405 (2009).

${ }^{57}$ A. A. Ignatov, K. F. Renk, and E. P. Dodin, Phys. Rev. Lett. 70, 1996 (1993).

${ }^{58}$ H. Kroemer, arXiv:cond-mat/0009311v1 [cond-mat.mes-hall].

${ }^{59}$ E. Mosekilde, R. Feldberg, C. Knudsen, and M. Hindsholm, Phys. Rev. B 41, 2298 (1990).

${ }^{60}$ E. Mosekilde, J. S. Thomsen, C. Knudsen, and R. Feldberg, Phys. D (Amsterdam) 66, 143 (1993).

${ }^{61}$ H. Ito and Y. Ueda, Phys. Lett. A 280, 312 (2001).

${ }^{62}$ T. Mausbach, T. Klinger, A. Piel, A. Atipo, T. Pierre, and G. Bonhomme, Phys. Lett. A 228, 373 (1997).

${ }^{63}$ A. E. Hramov and A. A. Koronovskii, Europhys. Lett. 80, 10001 (2007).

${ }^{64}$ K. Pyragas, Phys. Rev. E 56, 5183 (1997).

${ }^{65}$ A. E. Hramov, A. A. Koronovskii, and O. I. Moskalenko, Europhys. Lett. 72, 901 (2005).

${ }^{66}$ A. E. Hramov, A. A. Koronovskii, and M. K. Kurovskaya, Phys. Rev. E 78, 036212 (2008). 